\title{
APUNTES NECESARIOS ACERCA DE LA RELACIÓN ENTRE EJERCICIOS, PROBLEMAS Y TAREAS
}

\author{
Miguel Erasmo Zaldívar Carrillo e Israel Mayo Parra \\ Centro de Estudios de Ciencias de la Educación (CEDU), Cuba
}

\section{DESARROLLO}

Las definiciones de tareas, ejercicios y problemas han sido trabajadas, a lo largo de las últimas décadas, por muchos autores (Rubinstein S., 1985; Jungk W., 1982; Labarrare A., 1994; Lara D., 1995; Amat A., 1999; entre muchos otros). Uno de los aspectos en los que se ha ganado claridad está en las definiciones, clasificaciones y relaciones entre estos conceptos. Sin embargo, quedan aspectos por dilucidar.

Existen, por ejemplo, numerosas concepciones sobre las tareas docentes. N. E. Kuznetzova las concibe como "el medio para la dirección del proceso y procedimientos de la actividad, para el profesor y como medio para dominar los conocimientos y las habilidades para el alumno" (Citada por R. Deveza, 2000, p. 24).

D. B. Elkonin (1983) la define como "la unidad básica (célula) de la actividad docente, el medio que garantiza el estudio para los alumnos y el medio a través del cual los profesores crean las diferentes situaciones de enseñanza en la clase".

Por su parte C. Alvarez (1994) considera que las tareas docentes son la "célula del proceso docente, aquel proceso que se realiza en ciertas circunstancias pedagógicas, con el fin de alcanzar un objetivo de carácter elemental, de resolver el problema planteado al estudiante por el profesor".

Las definiciones anteriores abordan diferentes aspectos de lo que se entendería por tarea docente. La primera hace referencia a su propiedad de ser medio para la dirección del proceso y el dominio de los conocimientos tal posición nos perece correcta pero insuficiente. Abordar una propiedad esencial de un objeto o fenómeno nos permite un acercamiento a su esencia, pero solo eso, un acercamiento. En este sentido creemos que esta definición es insuficiente para dirigir el trabajo del maestro en clases por cuanto existen aspectos que no resultan abordados por el autor y que, en nuestra opinión, son esenciales.

Para Elkonin, en primer lugar, es la célula de la actividad docente. Tal afirmación, que generalmente se asume como la de significar su condición de elemento más simple, enriquece la anterior definición, si se interpreta con ello, que la actividad docente se desarrolla de tarea en tarea; que cada tarea tiene una dinámica propia, una relativa independencia y, que cumple su función solo dentro de lo que llamaríamos el sistema de tareas docentes. Si nos fijamos un poco, podría decirse que tal afirmación nos acerca a un modelo que explica cómo deben asumirse por el maestro y el estudiante el diseño, dirección, ejecución y control de las mismas. En tal sentido, pudiera decirse que dejar de realizar una tarea tiene un efecto que impacta mucho más allá de los aparentes límites de la misma.

La definición de Carlos Álvarez aporta más elementos de importancia a este análisis. Él las reconoce como célula básica del proceso y además las asume como un proceso. En este punto es bueno 
detenernos. Entenderlas como medio para, es, en nuestra opinión, una limitación, si asumimos el significado que el término medio tiene para la didáctica. No significa que no lo sea, sino, que como medio, al ser tarea adquiere una cualidad que la distingue, la de proceso. La tarea es proceso y debe generar procesos, si pierde esta condición deja de ser tarea; por lo menos como debe entendérsela en la actualidad. Debe entenderse, además, que los procesos generados por las tareas generan impactos en el alumno, el profesor y el grupo en general.

Para los autores de este trabajo resulta una limitación de la definición de Álvarez plantear "con el fin de alcanzar un objetivo de carácter elemental, de resolver el problema planteado al estudiante por el profesor". Ciertamente la tarea debe estar dirigida a un objetivo pero este no debe verse como la solución del problema planteado, dado que, por un lado, no toda tarea deviene problema, aspecto que trataremos más adelante, y por otro, la solución de una tarea puede y, debe, siempre que se pueda, generar otras tareas.

Partiendo de los conceptos analizados, los autores consideramos que entre los aspectos a destacar como esenciales deben estar:

- La tarea docente es la unidad de análisis de la clase, el elemento más simple de la relación maestro-alumno, del proceso de enseñanza-aprendizaje.

- La clase se constituye en una serie ordenada y planificada de tareas docentes conectadas entre sí con determinada intencionalidad.

- Implican la presentación de ejercicios, problemas, situaciones de aprendizaje y metas cuya consecución redunde en la apropiación de conocimientos (habilidades), modelos de actuación, valores y sentimientos por los alumnos y profesores.

- Son medios con cualidad de procesos que deben generar otros procesos en el alumno, el profesor y el colectivo.

Dentro de los estudiosos de las tareas docentes es comúnmente aceptado que la estructura de las mismas está dada por la orientación, la ejecución y el control, y que su contenido incluye la información, las acciones que el sujeto debe realizar, y los procedimientos intelectuales para su realización; lo que implica tener en cuenta la estructura y contenido de las tareas docentes, lo que resulta muy importante para que se pueda realizar el diagnóstico pedagógico del aprendizaje y la formación en general del alumno y el profesor.

Un aspecto que no debe olvidarse resulta de las transformaciones que hoy ocurren en la educación cubana en las enseñanzas primaria y secundaría básica. Si antes asumíamos que todos los polos que interactúan dentro del proceso pedagógico se transforman, o lo que es lo mismo todos enseñan y aprenden, como una aproximación de compromiso al carácter dialéctico del proceso pedagógico, en la actualidad debemos asumirlo como una realidad insoslayable que debe ser tenida en cuenta, dada la juventud e inexperiencia de una parte no despreciable de los que tienen la tarea de educar. 
También son variadas las clasificaciones de las tareas docentes. S. F. Zhuikov (1983) las clasifica en:

- Tareas para fijar el material estudiado.

- Tareas características del proceso de adquisición de conocimientos y habilidades.

Esta clasificación tiene la virtud de originar dentro de la clasificación de tareas, los espacios necesarios para que el maestro se desplace de la zona actual de desarrollo de sus estudiantes a la potencial. En tal sentido deberá entenderse que las tareas para fijar lo estudiado caen dentro del área actual de desarrollo generando los que llamaríamos ejercicios. Las segundas, caen dentro de la zona potencial de desarrollo traduciéndose en problemas.

Por su parte D. M. Grichin (ver Majmutov, 1983) las divide en :

- Cognoscitivas: las que presentan como característica fundamental el desconocimiento del resultado, con un conocimiento mayor o menor de los medios de su consecución

- Prácticas: la caracterizadas por un conocimiento con anterioridad del resultado con un medio desconocido para su consecución.

- Creativas: las que están dadas por el desconocimiento del resultado, así como del medio para llegar a él.

Uno de los problemas a los que se enfrenta el profesor al utilizar las disímiles clasificaciones que aparecen en la bibliografía especializada es el de entender cuál es la base clasificatoria sobre la que se realizan, fundamentalmente porque resultan, desde el punto de vista lógico formal, incorrectas. Una de las exigencias a la clasificación de conceptos está en que la suma de los volúmenes de los conceptos generados en las clases debe ser igual al volumen del concepto clasificado. Esta es una limitación de la clasificación anterior.

En general esta clasificación puede ser, bien utilizada por el docente, de mucha ayuda. La base clasificatoria tiene la virtud de partir del conocimiento o no por el estudiante de las vías y/o resultados de la tarea lo que obliga a profundizar en la utilización del diagnóstico de los estudiantes y a preparar la instrucción para que el alumno trabaje dentro de la zona potencial de desarrollo. Sin embargo, quedaron fuera de la clasificación las tareas en las que el estudiante conoce la vía ce solución y el resultado que alcanzará, las que comúnmente denominamos de nivel reproductivo; y que tienen una enorme importancia dentro del proceso docente educativo dado que resultan vía insustituible para la formación de las habilidades.

Bajo la denominación de tareas cognoscitivas, A. Pidkasisti y C. Rojas las clasifican en cuatro grupos:

- Por modelo: las que incluyen la totalidad de datos necesarios para realizarlas y el procedimiento a seguir en calidad de modelo de tarea.

- Reproductiva: las que requieren información obligatoria sobre su realización que el alumno convierte en procedimiento para su solución, para lo cual incorpora conocimientos y habilidades ya adquiridos. 
- Productiva: las que al resolverlas el alumno obtiene una nueva información sobre el objeto utilizando como instrumentos para ello conocimientos y procedimientos ya adquiridos.

- Creativas: las que implican una profunda aplicación por el alumno de sus conocimientos y procedimientos en situaciones nuevas que requieren la creatividad al desarrollar ellos sus propios razonamientos.

Todas estas clasificaciones resultan útiles para la planificación por el docentes de sus clases o sistema de clases, sin embargo, los autores consideran que resulta necesario tener en cuenta, además, lo que denominan tareas docentes (profesionales) integradoras de implicación personal, entendiendo por tales a aquellas tareas en que se modelan no sólo problemas o ejercicios que exigen la aplicación de conocimientos hábitos y habilidades, sino que también suponen la toma de posición en cuanto a problemas éticos relacionados con la vida presente o futura del sujeto.

Este tipo de tareas integra lo instructivo y lo educativo y tiene marcado carácter personológico, ya que compromete integralmente al educando, no sólo a su esfera intelectual.

Por lo discutido hasta aquí se entenderá que los problemas y ejercicios son tipos de tareas, osea, están incluidos en las clasificaciones dadas anteriormente. Respecto a la relación entre estos dos tipos de tareas se pueden hacer algunas consideraciones.

Algunos autores como Ball (1970) citado por Cruz M., 1997, al trabajar el concepto de problema, no aportan elementos suficientes para establecer sus límites con los de tareas y ejercicios. Lara D., (1995) y Amat A., (1999), reducen el concepto de tarea al concepto de problema. Se les debe señalar como aspecto positivo el énfasis en el carácter activo de ambos polos del proceso de enseñanza (el maestro y el alumno), elemento que está en correspondencia con el concepto de zona de desarrollo potencial o próximo, desde cuyas posiciones todos los polos que interactúan en el aprendizaje están sometidos a cambios, transformaciones, desarrollo.

Algunos autores, al establecer la diferencia entre ejercicio y problema, hacen referencia al desconocimiento de la vía de solución en este último para que se le pueda considerar como tal (Pérez F., 1995; Labarrere A., 1994). Aunque esta posición en principio resulta acertada, se debe tener en cuenta que existen muchas situaciones en las que una persona desconoce el camino o vía de solución y que no resultan problemas para él pedagógicamente hablando, aún suponiendo que desee resolverla, dado que no cuenta con las posibilidades personales (cognitivas e instrumentales) para ello. Con esto se quiere decir que, para la Pedagogía, no es suficiente, al definir problema, hacer referencia al componente objetivo y subjetivo del mismo en el sentido planteado. Los autores de este trabajo entienden que, para que un problema cumpla su función pedagógica deberá estar, además, ubicado en la zona potencial de desarrollo del sujeto que lo trata de resolver, o sea, que con determinados niveles de ayuda las pueda resolver.

Otros autores confunden alguno de los conceptos debatidos con el sistema de relaciones a que ellos deben conducir. Para Moya (1996) citado por Cruz M., (1997), por ejemplo, la tarea docente es "el sistema de relaciones que demanda la realización de determinadas acciones, encaminadas a transformar dicho sistema, apuntando hacia el descubrimiento de los vínculos que la sostienen" (Moya, 1996, pág. 10; citado por Cruz M., 1997). Se debe tener en cuenta que la tarea en sí no es un sistema de relaciones; una tarea bien planteada conduce a ello: a un sistema de relaciones que se puede analizar en varias 
dimensiones, las relaciones alumno-objeto de aprendizaje, las relaciones alumno-alumno y alumnoprofesor, etcétera.

Se debe asumir, en coincidencia con Labarrere, que "la tarea es una determinada situación en la cual existen nexos, relaciones, cualidades de y entre los objetos que nos son accesibles directamente o indirectamente a la persona" (Labarrere, 1994, pág. 19). Esta definición arroja luz a las diferencias que deben existir entre ejercicio y problema: cuando esos nexos, relaciones, son accesibles directamente a la persona se está haciendo referencia al concepto de ejercicio, o sea, el ejercicio demanda del sujeto la utilización de instrumentaciones y conocimientos que caen en el área de su zona actual de desarrollo; cuando son accesibles, solamente de manera indirecta, o sea, precisando ayuda, se esta haciendo referencia al concepto de problema. De manera que el problema debe caer, para considerarse didácticamente como tal, dentro de la zona potencial de desarrollo, de lo contrario, no cumpliría función alguna pues al irse fuera de sus posibilidades se torna insoluble por el sujeto.

\section{Clasificación de los problemas docentes}

Algunos autores al clasificar los problemas los han distinguido en abiertos y cerrados tomando como base de la clasificación la calidad de respuestas o vías de solución que admiten o exigen (ya sean de carácter lógico o creativo) (Bertoglia R., 1990; Gil P., 1997). Este último autor refiere que en los problemas cerrados, la solución se deduce en forma lógica a partir de la información que aparece en el planteamiento del problema y que resulta suficiente para encontrar la respuesta correcta. Según plantea, el resolutor dispone de toda la información, solo necesita integrarla aplicando los recursos de la lógica; por ello suelen llamarse problemas de inferencia lógica. En los abiertos, plantea, que el resolutor necesita ir más allá de la información recibida, utilizándola de manera distinta y/o modificando los significados atribuidos a los elementos del problema. Los recursos lógicos resultan ahora insuficientes, hay que apelar a la creatividad.

Coincidiendo con lo expuesto se debe hablar de la existencia de tareas abiertas o cerradas. Sin embargo, la clasificación anterior es insuficiente para orientar el trabajo de los maestros hacia el desarrollo de aspectos tan importantes como las particularidades del pensamiento y específicamente la fluidez y flexibilidad, de modo que es necesario ampliar la clasificación debatida.

Dada la necesidad de crear espacios para la estimulación del desarrollo de algunas particularidades individuales del pensamiento como la flexibilidad y la fluidez, resulta pertinente clasificar las tareas cerradas según sea la cantidad de respuestas o vías de solución que contengan y se exijan, en totalmente cerradas y semicerradas; coincidiendo ambas en el carácter lógico de las respuestas o vías de solución que contienen potencialmente y en que el resolutor dispone de toda la información necesaria y suficiente para resolverla (solo necesita integrarla aplicando los recursos de la lógica). Se diferencian en que las cerradas solo contienen o exigen una única vía de solución y, o respuesta posible, y las semicerradas contienen más de una. Debe comprenderse que el efecto de ambos tipos de situaciones en el desarrollo de la fluidez y la flexibilidad del estudiante y de su pensamiento en general no es el mismo.

Por otro lado las tareas abiertas deben ser clasificadas análogamente según sea la cantidad de respuestas creativas que se exijan en semiabiertas, las que exigen al resolutor una única solución creativa, $y$ abiertas, las que exigen al resolutor varias soluciones creativas. Aquí se han asumido los términos de abierto y semiabierto en el sentido convencional que se declara. 
Este tipo de clasificación será fundamental para poder dar tratamiento, en clases, al desarrollo de algunas particularidades del pensamiento como la fluidez y la flexibilidad.

Llegado a este punto cabría preguntarse ¿cómo implementar en el sistema de clases las tareas docentes?.

Exigencias metodológicas para la implementación en la clase de las tareas docentes

Por último los autores considera que resulta necesario tener en cuenta algunas exigencias para que la utilización de las tareas docentes redunde en mayor calidad para la clase. Ellas son:

- Organización sistémica. Las tareas deben guardar nexos entre sí. Unas deben ser condiciones previas para la realización de otras.

- Complejidad ascendente. Las tareas docentes deben estar gradadas de acuerdo a su nivel de complejidad, de modo que su realización por el alumno implique el aumento gradual y controlado de las exigencias didácticas y educativas que se le plantean. En estas condiciones, cuando el alumno se detiene en una tarea de un nivel, esto facilita el diagnóstico de la dificultad de aprendizaje o educativa que se manifiesta.

- Integradoras. Las tareas en su construcción deben incluir situaciones, ejercicios, problemas, etc., que obliguen al alumno a utilizar conocimientos de otras asignaturas, manifestar sus opiniones, sentimientos y valores a través de la toma de posición. Es decir deben ser integradoras de lo curricular y lo formativo.

- Versátiles. Las tareas deben tener diversidad en su presentación, facilitando con ello la atención a la diversidad y posibilitando su empleo no sólo en la clase, sino también en el trabajo independiente extraclase.

Estas consideraciones se han elaborado en la práctica, como resultado del trabajo metodológico realizado en el centro que los autores dirigen. Se ha podido apreciar que a los maestros les ha resultado mas orientador poseer una concepción para la selección y estructuración del contenido de la clase que dé respuestas a las múltiples demandas que en la actualidad la calidad de la educación les plantea.

\section{BIBLIOGRAFÍA}

ALVAREZ DE ZALLA C. La escuela en la vida. Edit. Academia, 1994

AMAT, A. M. (1999). Una alternativa metodológica basada en la resolución de ejercicios para contribuir al desarrollo del pensamiento lógico de los estudiantes de Secundaría Básica a través de la enseñanza de la Matemática. Tesis en opción al título académico de Master en Didáctica de la Matemática. Holguín. ISP "José de la Luz y Caballero".

ARTEMieVA, T. El aspecto metodológico del problema de las capacidades. Edit. Pueblo y Educación, 1985.

BertogliA, R. L. (1990). Psicología del aprendizaje. Universidad de Antofagasta, Chile.

CóRDOVA, M. La estimulación intelectual en el curriculum regular. En proceso de edición. 
CRUZ, R. M. (1997). Estrategia para la elaboración de ejercicios del Análisis Diofántico. Tesis en opción al título académico de Master en Didáctica de la Matemática. Facultad de Ciencias. Departamento de Matemática Computación. ISP José de la Luz y Caballero. Holguín. Cuba.

DEBONO, E. Aprender a pensar. Impresión ligera. Facultad de Economía. Universidad de la Habana. 1980.

GIL, P. (et-al) (1996). Temas escogidos de la didáctica de la Física. Editorial Pueblo y Educación. Ciudad de la Habana. Cuba. 122p.

GLASER, R. On the nature of expertice. En Human memory and cognitive capabilities,. North Holland. Elser View Science publisher 1986.

GONZÁLEZ, F. Y MITJÁNS, A. La personalidad, su educación y desarrollo. Edit. Pueblo y Educación.1989 .

JUNGK, W. (1982). Conferencias sobre metodología de la enseñanza de la Matemática II. Primera parte.

LABARRERE, A. Metacognición y capacidad cognitiva. Impresión ligera. La Habana 1989.

LABARRERE, S. A. (1996). Pensamiento. Análisis y autorregulación de la actividad cognoscitiva de los alumnos. Editorial Pueblo y Educación. La Habana. Cuba. 101 p.

LARA, D. M. (1995). Sistema de tareas didácticas para la dirección del trabajo independiente en la metodología de la enseñanza de la Física. Tesis en opción al grado científico de Doctor en Ciencias Pedagógicas. ISP Enrique José Varona. Ciudad de la Habana. Cuba.

MAJMUtov, M. I. (1983). La enseñanza problémica. Editorial Pueblo y Educación. Ciudad de la Habana. Cuba. $349 \mathrm{p}$.

MASLOW, A. Some educational implications of humanistic psichologies in Harvard Educational Review. No 38, 1968.

MAYER, R. Pensamiento, resolución de problemas y cognición. Edit. Paidós. 1986.

PÉREZ, F. (1995). La formulación y solución de problemas en la enseñanza de las asignaturas de Ciencias Naturales. Curso Evento Pedagogía '95. La Habana. Cuba.

RODRíGUEZ DEVEZA, R. Las tareas docentes como medio diagnóstico y formativo de la generalización del pensamiento en el aprendizaje de la gramática inglesa. Tesis de maestría, inédita, ISPH. 2000

ROGERS, C. Libertad y Creatividad en Educación en los 80. Edit Paidós. 1991.

RUBINSTEIN, S. L. (1985). El pensamiento y los caminos de su investigación. Editorial Progreso. Moscú.

STENBERG, R. La inteligencia humana. Vol 1 y 2 Barcelona. Edit. Paidós 1982.

VIGOTSKY, L. Historia del desarrollo de las funciones psíquicas superiores. Edit. Científico-Técnica. C. Habana. 1987.

ZALDíVAR, C. M. (2001). El desarrollo de la flexibilidad del pensamiento a través del proceso de enseñanzaaprendizaje de la Física en el nivel medio superior. Tesis presentada en opción al título académico de Master en Investigación Educativa, ISP “José de la Luz y Caballero”, Holguín, 2001. 


\title{
Contactar
}

Revista lberoamericana de Educación

\author{
Principal OEI
}

